\title{
Time/frequency coding for Brillouin distributed sensors
}

\author{
Sébastien Le Floch*a ${ }^{\text {a }}$ Florian Sauser ${ }^{\mathrm{a}}$, Marcelo A. Soto ${ }^{\mathrm{b}}$, Luc Thévenaz ${ }^{\mathrm{b}}$ \\ ${ }^{a}$ HE-ARC, Rue de la Serre 7, CH-2610 Saint Imier, Switzerland; \\ ${ }^{b}$ EPFL, Swiss Federal Institute of Technology, Institute of Electrical Engineering, \\ SCI-STI-LT Station 11, CH-1015 Lausanne, Switzerland \\ *sebastien.lefloch@he-arc.ch; phone +41 3293022 49; fax +41 3293022 04; www.he-arc.ch
}

\begin{abstract}
In this paper, we propose a novel coding for long range Brillouin Optical Time Analysis (BOTDA) distributed sensors based on a combination of time and frequency pulses, resulting in an additional coding gain of $\sqrt{2}$ with respect to traditional intensity-modulated codes. The generation of frequency-chirped pseudo-arbitrary pulses in return-to-zero (RZ) format with a Direct-Digital Synthesizer (DDS) is presented and the coding gain is experimentally verified, perfectly matching its theoretical value.
\end{abstract}

Keywords: Stimulated Brillouin scattering, fiber-optics sensors, optical signal processing, distributed fiber sensors.

\section{INTRODUCTION}

Distributed fiber sensors based on stimulated Brillouin scattering for long distance measurements have been deeply studied for two decades, as they make possible accurate temperature/strain measurements over tens of kilometers with a metric spatial resolution ${ }^{1,2}$. Research on Brillouin optical time-domain analysis (BOTDA) reached a new level during the past three years thanks to pulse coding techniques. Simplex code was first employed to increase the distance range up to $50 \mathrm{~km}$ with a spatial resolution of one meter ${ }^{3}$. Non return to zero (NRZ) coding causes strong distortions on the local Brillouin gain spectra, due to the pre-excitation of the acoustic wave leading to a distorted response ${ }^{4}$. In this paper, we introduce a unique and original coding scheme named "time/frequency code", specially configured and optimized for BOTDA sensing. Each pump pulse of the coded sequence has a distinct frequency that is generated at a very fast rate by a direct digital synthesizer (DDS) in the microwave domain. A general principle of the code is presented, and distributed measurements are demonstrated over a distance range of $25 \mathrm{~km}$ with a spatial resolution of 2 meter.

\section{TIME/FREQUENCY CODE}

So far coding schemes in BOTDA are based on the on/off intensity modulation of pump pulses. For example, the second sequence of the Simplex code of length $L=15$ is 011001100110011 . Considering globally all sequences, there is on average the same number of " 0 " and " 1 " in this code. The decoding process shows no distortion only if all " 1 " pulses carry the same energy and, since all sequences are distinct, this requires that the boosting amplification on the pump pulses is absolutely temporally flat and invariant from sequence to sequence ${ }^{3}$. Together with the serious limitation due to the onset of other nonlinear effects ${ }^{5}$ (modulation instability, forward Raman scattering), this practically limits the peak pump power to approximately $15-20 \mathrm{dBm}$. Actually, since this peak power is strictly limited, a binary intensity coding is not using the full energy potentiality of the sequence, since no energy is transmitted $50 \%$ of time - during the " 0 " bits - and this automatically reduces the gain in signal-to-noise ratio resulting from the coding.

The invented code presented here is filling these energy temporal gaps by realizing coding sequences of frequency modulated pulses having all the same peak power. This way the full energy potentiality of the sequence is exploited and the frequency coding is arranged, so that it corresponds to the frequency grid used for scanning the pump-probe frequency difference. So, instead of sending interacting pump and probe signals with a fixed sequentially incremented frequency difference, a distinct frequency is allocated to each pump pulse forming the code and each sequence presents a different arrangement of these frequency-modulated pulses. Once all traces are recorded, the equivalent traces as those produced by a very intense single frequency pulse can be retrieved via a consequent data processing based on matrix 
calculations. The result is an additional gain in SNR of $\sqrt{2}$ with respect to binary intensity codes, corresponding to the 2fold increase in energy contained in the sequence.

This BOTDA-dedicated time-frequency code is built following this principle: let assume a code length of $L=3$, so containing 3 distinct frequencies. At each position along the fiber, there are 3 unknown quantities: the amount of Brillouin gain for each of these 3 frequencies. The total number of unknown quantities is then $\mathrm{L}^{2}$, which means that $\mathrm{L}^{2}$ experiences are necessary to retrieve all the local Brillouin gains. For the construction of the coding matrix, shown in Fig.1, we create an identity matrix of rank 3 , corresponding the upper left $3 \times 3$ block in blue. A second matrix, which is a rotation of the identity matrix, is juxtaposed (red), and then there is a null matrix (black), otherwise the matrix would be singular. This creates a matrix of rank $\mathrm{L} \times \mathrm{L}^{2}$. The full coding matrix is made such that it constitutes a block-circulant matrix with circulant blocks (BMCB). Figure 1 shows the corresponding matrix for $\mathrm{L}=3$ (rank $\mathrm{L}^{2}$ ). In fact, it corresponds to a 3 dimensional coding (Brillouin gain at each position and each frequency), but represented in 2 dimensions.

\begin{tabular}{|c|c|c|c|c|c|c|c|}
\hline \multicolumn{3}{|c|}{$\overbrace{}^{\text {Pos.1 }}$} & \multicolumn{2}{|c|}{ Pos.2 } & \multicolumn{3}{|c|}{$\overbrace{}^{\text {Pos. }}$} \\
\hline & 0 & 0 & $\begin{array}{ll}0 & 1\end{array}$ & 0 & 0 & 0 & \\
\hline 0 & 1 & 0 & $\begin{array}{ll}0 & 0\end{array}$ & 1 & 0 & 0 & 0 \\
\hline 0 & 0 & 1 & 10 & 0 & 0 & 0 & 0 \\
\hline 0 & 0 & 0 & 10 & 0 & 0 & 1 & 0 \\
\hline 0 & 0 & 00 & $\begin{array}{ll}0 & 1\end{array}$ & 0 & 0 & 0 & 1 \\
\hline 0 & 0 & 00 & 00 & 1 & 1 & 0 & 0 \\
\hline 0 & 1 & 00 & 00 & 0 & 1 & 0 & 0 \\
\hline 0 & 0 & 10 & 00 & 0 & 0 & 1 & 0 \\
\hline & 0 & & 0 & & & & \\
\hline
\end{tabular}

Figure 1: Generating matrix of the time/frequency code with code length $\mathrm{L}=3$. Pos. stands for position; blue matrix is the identity matrix; red matrix is a rotate right matrix of the identity matrix; black matrix is the null matrix.

Each matrix line corresponds to a code sequence and the allocated frequencies for each pulse in the sequence are determined using the following rule: each group of $L=3$ elements on a line, corresponds to a pulse (or position) in the sequence, as shown in Fig.1. Each group contains at most one "1" and the position of this "1" in the group determines the allocated frequency. For instance, in the first line, the first group allocates frequency 1 to the sequence position 1 , then frequency 2 to position 2, then no light to position 3 (pulse is turned off). The second line allocates frequency 2 to position 1, then frequency 3 to position 2, then no light to position 3, etc... It was found that, by optimizing the variance when searching for the inverse matrix, this can be generalized to any $L$ being a prime number. For a code length $L$, theoretical considerations prove that the signal-to-noise improvement is $(\mathrm{L} / 2)^{1 / 2}$ compared to the standard pump-pulse technique. This is approximately $\sqrt{2}$ better than conventional intensity-based codes such as Simplex and complementary Golay codes. The decoding process is based on the following theorem ${ }^{6}$ : the inverse matrix of a non-singular BMCB is also a BMCB. As a result, the product of the inverse matrix with the matrix of data is equivalent to a correlation, so that Fast Fourier Transforms are used to perform the data processing ${ }^{7}$. Calculations require 2 minutes on a standard PC for a code length $L=199$ and 50kpts along the fiber, which is still somehow shorter than the acquisition time in long range BOTDA systems for a scan over 199 frequencies.

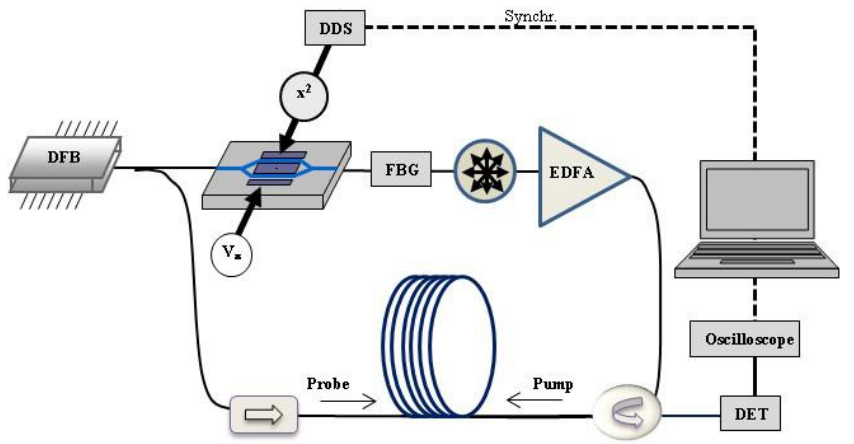

Figure 2: Experimental set-up. DDS: Direct-Digital Synthesizer; FBG: Fiber Bragg Grating; EDFA: Erbium doped fiber amplifier; DET: detector; DFB: distributed feedback laser. 


\section{EXPERIMENTAL SET UP}

Figure 2 shows a scheme of the experimental set-up. The output of a distributed feedback (DFB) laser source, operating at $1533 \mathrm{~nm}$ and delivering a $10 \mathrm{dBm}$ optical power, is split in two arms by an optical coupler. The continuous wave $(\mathrm{CW})$ probe, directly tapped from the laser source, propagates through the lower arm, where the sensing fiber is placed, and is then detected after a circulator. In the upper arm, an intensity modulator (IM), operating in suppressed carrier mode, is driven by a DDS frequency-agile microwave generator: the RF code sequences are uploaded into the DDS, its output is then frequency-doubled and fed to the IM. One of the two generated frequency sidebands is filtered out by a fiber Bragg grating to only leave the upper sideband, which is then amplified by an EDFA. A polarization scrambler is used to suppress the dependence on polarization of the Brillouin response along the fiber.

\section{MEASUREMENTS AND RESULTS}

In order to evaluate the coding SNR gain, we have performed a distributed measurement over $25 \mathrm{~km}$ of a standard G.652 fiber. The spatial resolution was set to $2 \mathrm{~m}$, corresponding to pump pulse duration of $20 \mathrm{~ns}$ (the DDS is able to deliver a distinct frequency at a $4 \mathrm{~ns}$ rate), in a RZ format: the zero segment duration was set to $60 \mathrm{~ns}$, leaving enough time to the acoustic wave to sufficiently damp ${ }^{4}$. The code length was $L=151$ (prime number), each trace being averaged 4 times, corresponding to a total theoretical SNR enhancement of about 200 (including time-averaging and the coding gain). The 151 distinct frequencies were equally spaced between $10.88 \mathrm{GHz}$ and $11.075 \mathrm{GHz}$, with a corresponding frequency step of $1.3 \mathrm{MHz}$. The pump and probe powers were $20 \mathrm{dBm}$ and $-10 \mathrm{dBm}$ respectively, this latter being set so that pump depletion is not exceeding $20 \%$ at the fiber output ${ }^{8}$. A key point to mention is that the intensity spatial profile of the distributed Brillouin gain appears nearly identical for all traces, since all sequences of pump pulses contain all frequencies, and in particular those that lie in the Brillouin gain spectrum (figure 3).

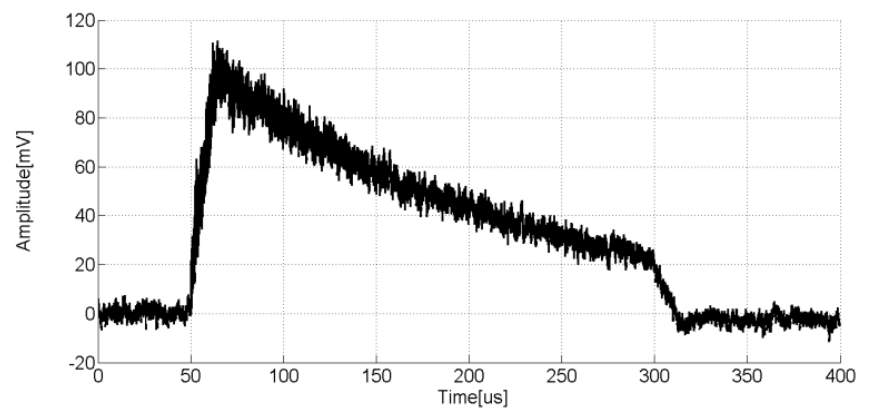

Figure 3: BOTDA intensity temporal profile obtained using a typical coded pulse sequence $(\mathrm{L}=151)$.

The variation in intensity between all the $\mathrm{L}^{2}$ traces was $\pm 20 \%$ for this measurement. Figure 4 shows the measurement of the Brillouin gain spectrum at the output of the fiber. The obtained residual standard deviation after a quadratic fitting, results in an experimental coding gain of $9.7 \mathrm{~dB}$, which is in agreement with the theoretical value $(9.4 \mathrm{~dB})$.

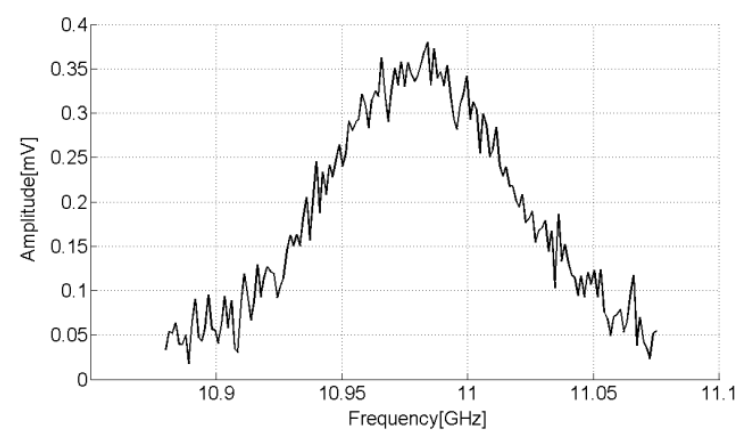

Figure 4: Brillouin gain spectrum at a distance of $24.5 \mathrm{~km}$, obtained after processing using the complex decoding procedure.

Finally, figure 5 shows the measured Brillouin frequency shift (BFS) along the fiber, with an accuracy of $\pm 1.5 \mathrm{MHz}$ at the end of the fiber. These results are of course still below the state-of-the-art, but the purpose of the experimental test 
was to demonstrate that the information can be securely retrieved, in spite of a complex decoding, and the coding brings the SNR enhancement that is anticipated. In addition this clearly proves that the coded frequencies are correctly generated in the DDS, with excellent accuracy (in fact better than $0.1 \mathrm{MHz}$ ). The next step will be the implementation of this code in a top performing BOTDA set-up.

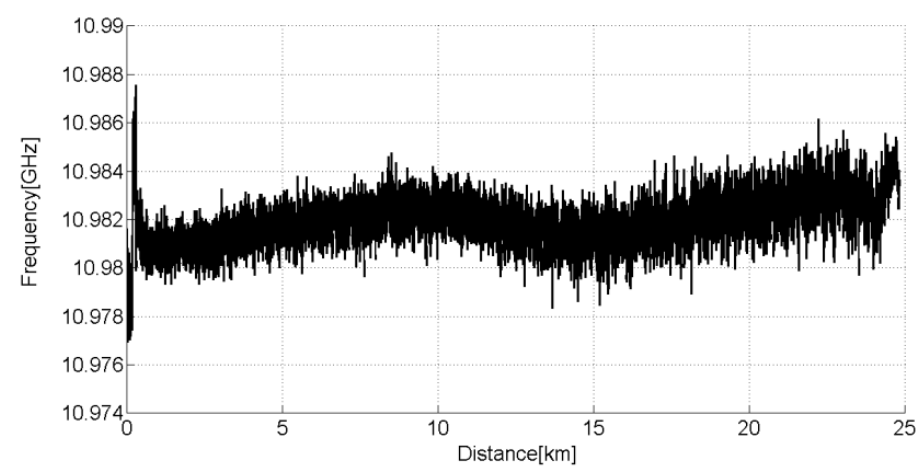

Figure 5: BFS in the fibe using time/frequency coding $(\mathrm{L}=151)$ with RZ pulses.

\section{CONCLUSION}

In conclusion, we have successfully shown that a specific dedicated coding - the time/frequency code - can be implemented in BOTDA sensors, taking fully advantage of the double scanning (position and pump-probe detuning) acquisition required for such sensors. The position and frequency scans are both rearranged by the coding, leading to a beneficial gain in SNR and the information can be reliably retrieved despite the complex array governing the rearrangement of the launched sequences. The direct digital synthesizer is a crucial element since its recent availability in the microwave domain makes possible the fast generation of short pulses, at distinct frequencies and at a rate in the nanosecond range to build the coded sequences. Measurements have proved that the coding gain follows the theoretical relation $(\mathrm{L} / 2)^{1 / 2}$, which is larger than conventional binary intensity-based codes.

\section{ACKNOWLEDGMENTS}

The authors would like to acknowledge the company OMNISENS (Switzerland), the Swiss National Foundation (Project NCCR-QP-IPP) and the Swiss Commission for Technology and Innovation (Project 13122.1) for their support.

\section{REFERENCES}

[1] Horiguchi, T., and Tateda, M., "BOTDA - non-destructive measurement of single-mode optical fibre attenuation characteristics using Brillouin interaction: theory", J. Lightwave Technol. 7, 1170-6 (1989).

[2] Horiguchi, T., Kurashima, T., and Tateda, M., "A technique to measure distributed strain in optical fibers", IEEE Photon. Technol. Lett. 2, 352 (1990).

[3] Soto, M. A., Bolognini, G., Di Pasquale, F. and Thévenaz, L., "50 km Simplex-coded BOTDA sensor for distributed strain and temperature measurements with $1 \mathrm{~m}$ spatial resolution", Optics Letters 35(2), 259-261 (2010).

[4] Soto, M. A., Bolognini, G. and Di Pasquale, F., "Analysis of pulse modulation format in coded BOTDA sensors", Optics Express 18(14), 14878-14892 (2010).

[5] Foaleng, S. M. and Thévenaz, L., "Impact of Raman scattering and modulation instability on the performances of Brillouin sensors", Proc. SPIE 7753, 77539V-1 (2011).

[6] Jiang, Z, Xu, Z. and Gao, S., "Algorithms for finding the inverses of factor block circulant matrices", Numerical Mathematics 15(1), 1-11 (2006).

[7] Vogel, C. R., "Computational methods for inverse problems", Frontiers in Applied Mathematics (2002).

[8] Thévenaz, L., Foaleng, M. F. and Lin, J., "Impact of pump depletion on the determination of the Brillouin gain frequency in distributed fiber sensors", Proc. SPIE 7753, 775322 (2011). 